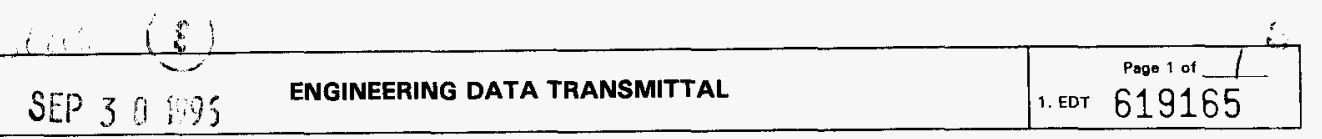

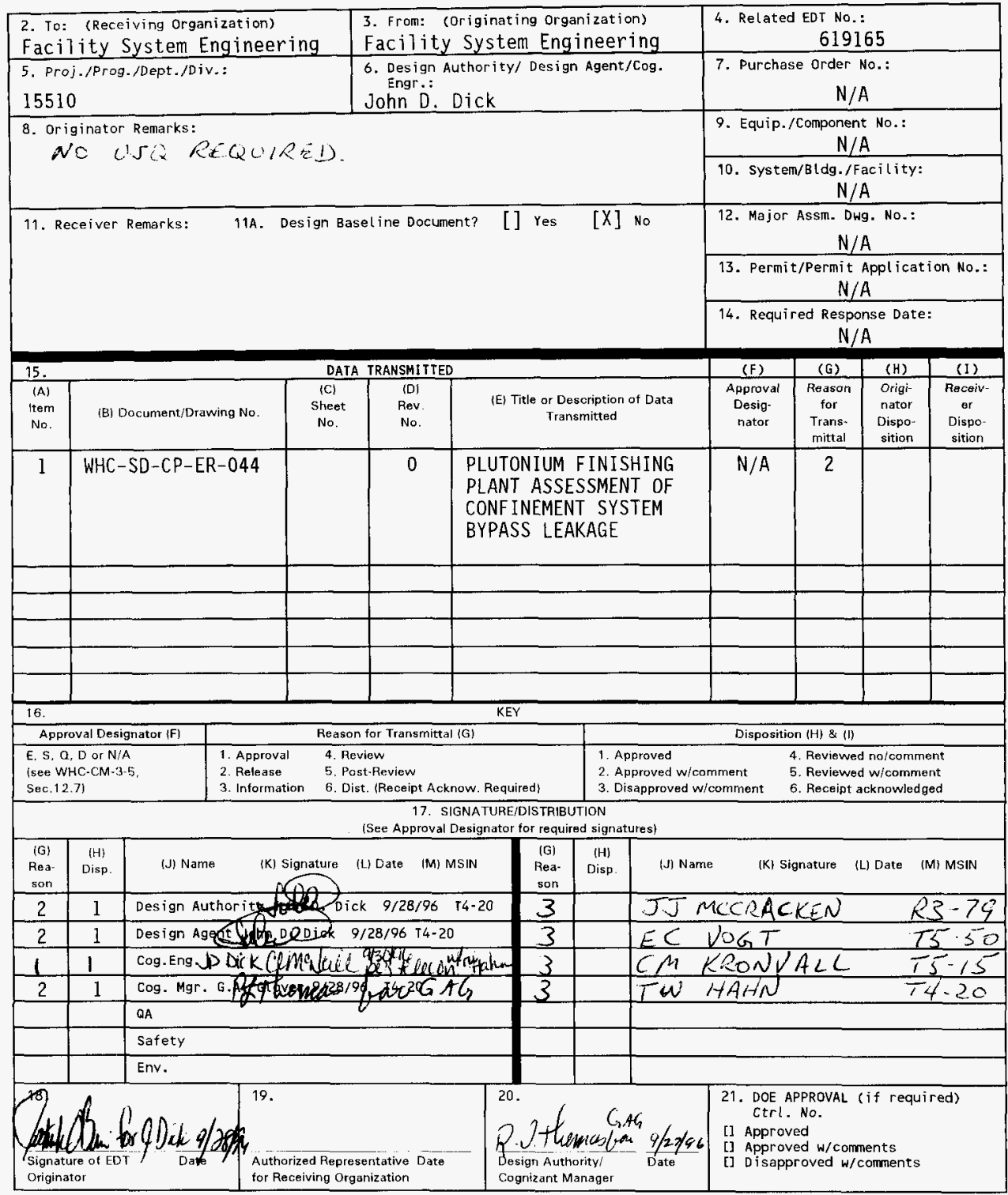

BD - 7400-172-2(05/96) GEF097 


\section{PLUTONIUM FINISHING PLANT ASSESSMENT OF CONFINEMENT SYSTEM BYPASS LEAKAGE}

John D. Dick

Westinghouse, Richland, WA 99352

U.S. Department of Energy Contract DE-AC06-87RL10930

$\begin{array}{lll}\text { EDT/ECN: } & 619165 & \text { UC: } 2050 \\ \text { Org Code: } & 15510 & \text { Charge Code: } \\ \text { B\&R Code: } & \text { EW7002010 } & \text { Total Pages: } 31\end{array}$

Key Words: HVAC, CONFINEMENT, BYPASS

Abstract: The purpose of this report is to document walk-through's of the safety class confinement systems at the Plutonium Finishing Plant (PFP). In addition this document outlines the actions taken to assess the confinement system for bypass leakage as well as establishing disposition for discovered deficiencies at the PFP.

TRADEMARK DISCLAIMER. Reference herein to any specific comercial product, process, or service by trade name, trademark, manufacturer, or otherwise, does not necessarily constitute or imply its endorsement, recommendation, or favoring by the United States Government or any agency thereof or its contractors or subcontractors.

Printed in the United States of America. To obtain copies of this document, contact: WHC/BCS Document Control Services, P.O. Box 1970, Mailstop H6-08, Richland WA 99352, Phone (509) 372-2420; Fax (509) 376-4989.
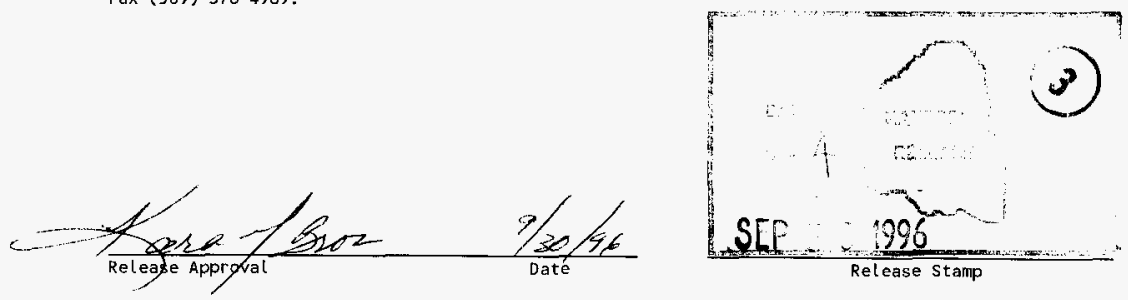

\section{Approved for Public Release}




\section{WHC-SD-CP-ER-044 REV 0 \\ PLUTONIUM FINISHING PLANT}

ASSESSMENT OF CONFINEMENT SYSTEM BYPASS LEAKAGE

2 


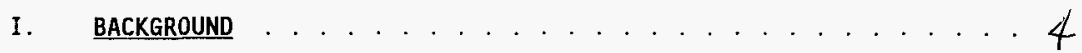

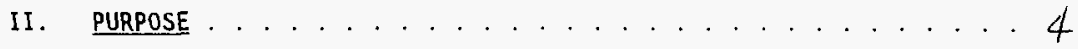

III. ENGINEERING ASSESSMENTS . . . . . . . . . . . . . . . . . 4

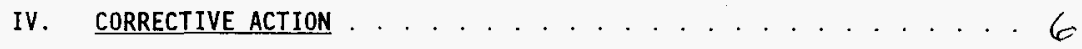

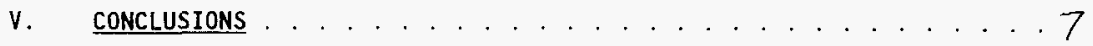

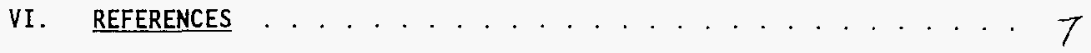

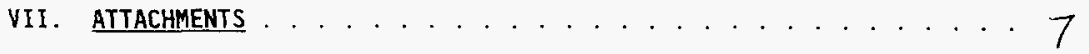




\section{BACKGROUND}

On March 15, 1996, the Department of Energy (DOE) issued a response to the Defense Nuclear Facilities Safety Board the "Plutonium Ventilation System Study Report" dated February 1996. One of the conclusions of the report was to perform a more detailed assessment of ventilation system bypass leakage and acceptance testing for safetyclass ventilation systems at our facilities. "Bypass leakage" refers to the potential for unfiltered exhaust from safety-class confinement systems, during either normal operation or accident conditions.

In response to the report, the DOE committed to perform walk-throughs of safety-class confinement systems to find any untested bypass leakage paths, analyze them and either take action to ensure that leakage testing includes these paths in the future or document why the facility would remain within its authorization basis if such leakage testing were not pertormed.

\section{PURPOSE}

The purpose of this report is to document walk-through's of the safety class confinement systems at the Plutonium Finishing Plant (PFP). In addition this document outlines the actions taken to assess the confinement system for bypass leakage as well as establishing disposition for discovered deficiencies at the PFP.

\section{ENGINEERING ASSESSMENTS}

The consensus standard currently used to assess the performance of the PFP nuclear air cleaning system is the American Society of Mechanical Engineers (ASME) standard N510. This standard covers both duct damper bypass testing and system bypass testing.

\section{REQUIREMENT}

Duct Damper Bypass Testing (ASME N510 section 12): This section involves leakage through closed dampers or valves intended to eliminate flow through duct work designed to bypass a HEPA filter air cleaning system. 


\section{ENGINEERING ASSESSMENTS (con't)}

\section{ASSESSMENT}

During the a self assessment of PFP ventilation system completed in September, 1995, a possible bypass leak vulnerability was discovered. This vulnerability consisted of the ability to route air from open face hoods located in room 159 around exhaust HEPA filters (see attachment 1). Due to system's configuration it is impossible to perform a meaningful bypass damper test.

\section{REQUIREMENT}

System Bypass Testing (ASME N510 section 13): This section focuses on other bypass routes associated with systems using HEPA filters that may contain bypass dampers, ducts, conduits, floor drains pipe penetrations, ect., which could potentially defeat the purpose of high efficiency nuclear air treatment components.

\section{ASSESSMENT}

The PFP Final Safety Analysis Report, WHC-SD-CP-SAR-021 states leakage through unfiltered pathways would occur if the facility became pressurized. The safety analysis also states that several events must occur for a discharge to the atmosphere to occur. These events include a damper supplying ventilation air to an area housing confinement vessels must have failed; loose materials is present in the glovebox or hood; there must be a breach of the glovebox or hood large enough so that the confinement structure or ventilation cannot maintain an adequate differential pressure across the breach to maintain contamination control; and, finally, the flow path to the existing exhaust system must be incapable of sweeping all the materials into the exhaust duct such as would occur if the wall to the outside had opened a large hole. Without detailed examination it is clear that such a series of independent events has a very low probability when combined with the low onsite/offsite consequences of plutonium spills into rooms postulated in WHC-SD-CP-SAR-021, Sections 9.1.1 through 9.1.4. 
Walls that separate radioactive material confinement zones from the atmosphere and nonradioactive material zones are considered safety class design features per the PFP Operational Safety Requirements (OSR) WHC-SD-CP-OSR-010, appendix B. These design features are maintained by configuration management procedures established in manuals WHC-CM-6-1 (Standard Engineering Practices) and WHC-CM-5-8 (Plutonium Finishing Plant Administration).

A walkdown of the PFP complex confinement features was completed July 8,1996 . The walkdown consisted of a visual inspection of ventilation/structural barriers between radiative marerial zones, non-radioactive material zones and atmosphere. The inspection revealed some minor air leakage paths around existing conduits, pipe, air ducts and door seals. Attachment 2 of this report lists deficiencies discovered during the inspection.

\section{CORRECTIVE ACTION}

To comply with requirements outlined in ASME N510 sections 12 and 13 the following actions were taken:

- Open face hoods in Room 159 were removed from service and the damper locked and tagged closed until the duct work between filter box FB-9AB and the stack manifold could be removed (see redline section of $\mathrm{H}-2-99531$ sheet 1 attached). This duct was removed April 30, 1996 as part of project 94-1.

- Work authorization 2Z-96-2009 has been initiated to correct the minor deficiencies listed in attachment 2 of this report. In addition, as a good maintenance practice, procedure ZSR-25-100 was established to provide annual ventilation zone barrier surveillance and insure compliance of safety class design feature requirements of the OSR. 


\section{CONCLUSIONS}

In conclusion, once the bypass route between filter box FB-9AB and the Stack Manifold was removed the possibility of bypassing a safety class filtration systems within PFP no longer exists. Therefore, the consensus standard ASME N510, section 12 has been meet.

The PFP safety analysis includes system bypass leakage consideration in the accident scenarios established in chapter 9 of the FSAR. Structural and ventilation confinement is a safety class design feature that require formal documentation in order to modify. However, in order to insure conformance to design procedures and identify deterioration of a structural component and/or door seals a annual surveillance was initiated. Therefore, the intent of the consensus standard, ASME N510 Section 13 have been meet.

\section{REFERENCES}

1) DOE EM-60 memo to RL dated May 3, 1996 (Reference 961533.5B)

2) Plutonium Ventilation System Study Report, dated February, 1996

3) Letter from O'leary to Conway dated March 15, 1996

4) ASME Standard N510, sections 12 and 13

5) PFP Satety Analysis Report WHC-SD-CP-SAR-021, dated 03/03/95

6) RL letter to WHC 96-TPD-65

\section{ATTACHMENTS}

1) WHC drawing H-2-99531, sheets $1-5$

2) BYPASS LEAKAGE INSPECTION DEFICIENCIES

3) PFP Safety Envelope (structural and ventilation confinement) Procedure ZSR-25-100 
WHC-SD-CP-ER-044 REV. 0

WHC drawing H-2-99531, sheets 1-5

ATTACHMENT 1 


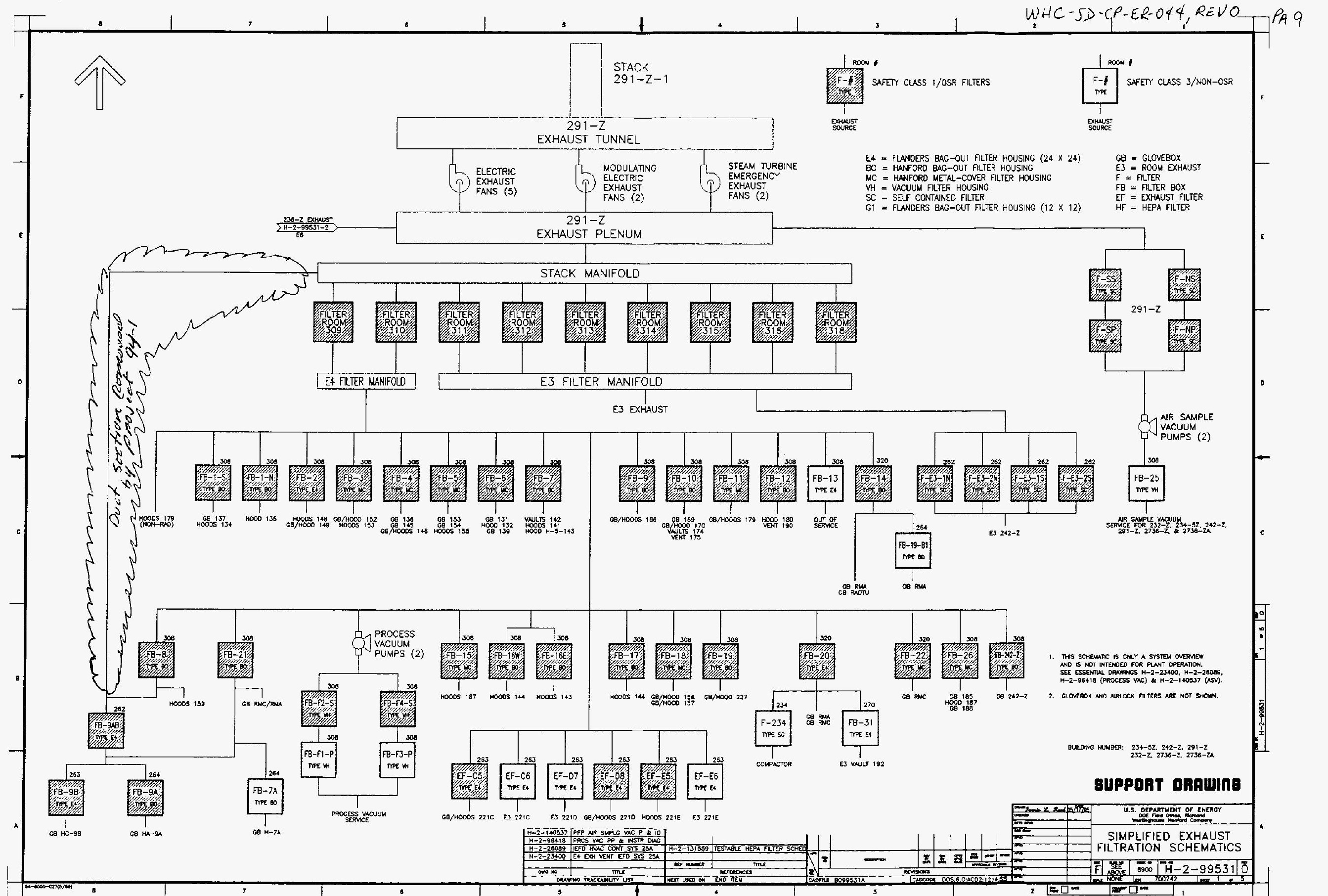




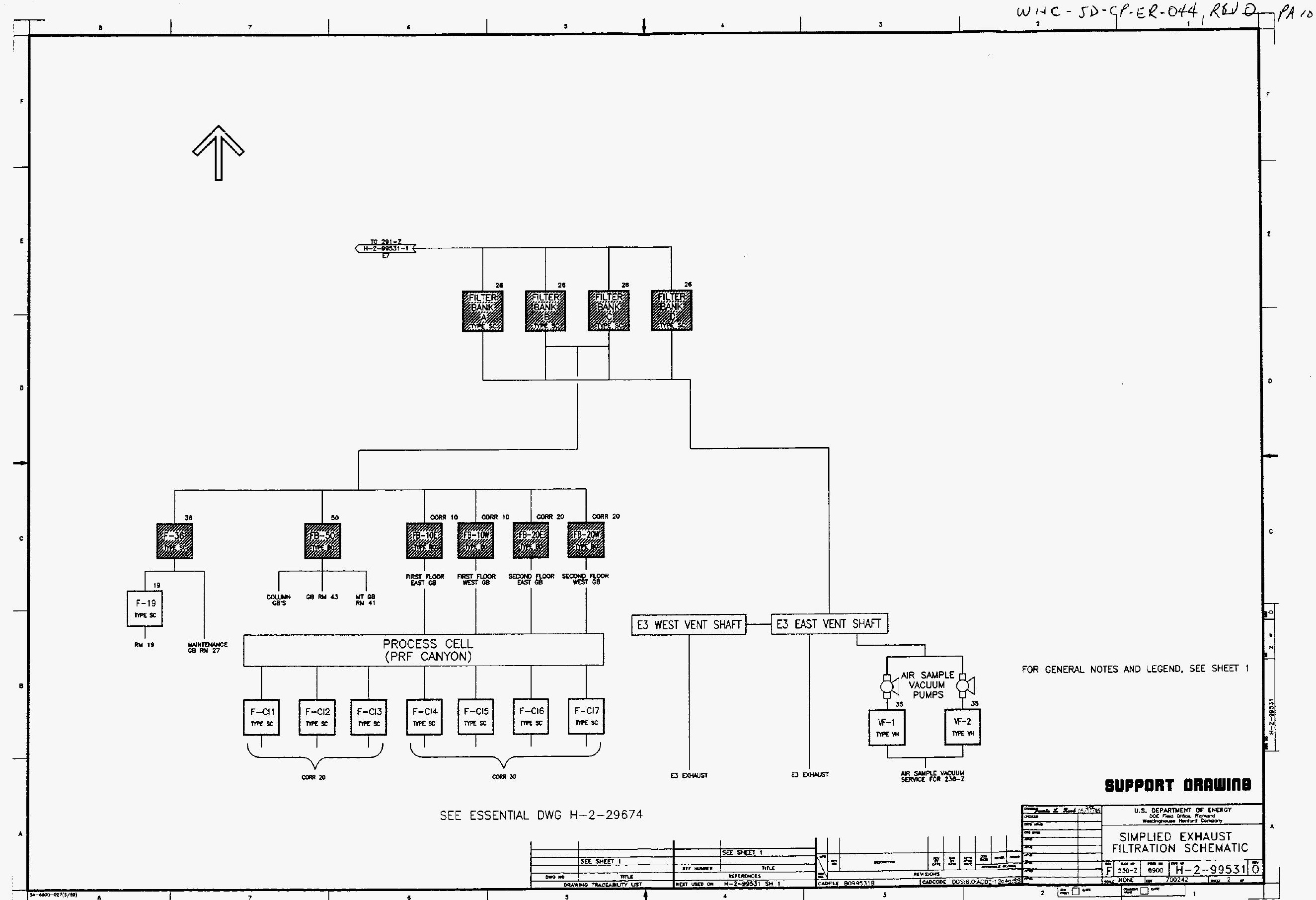




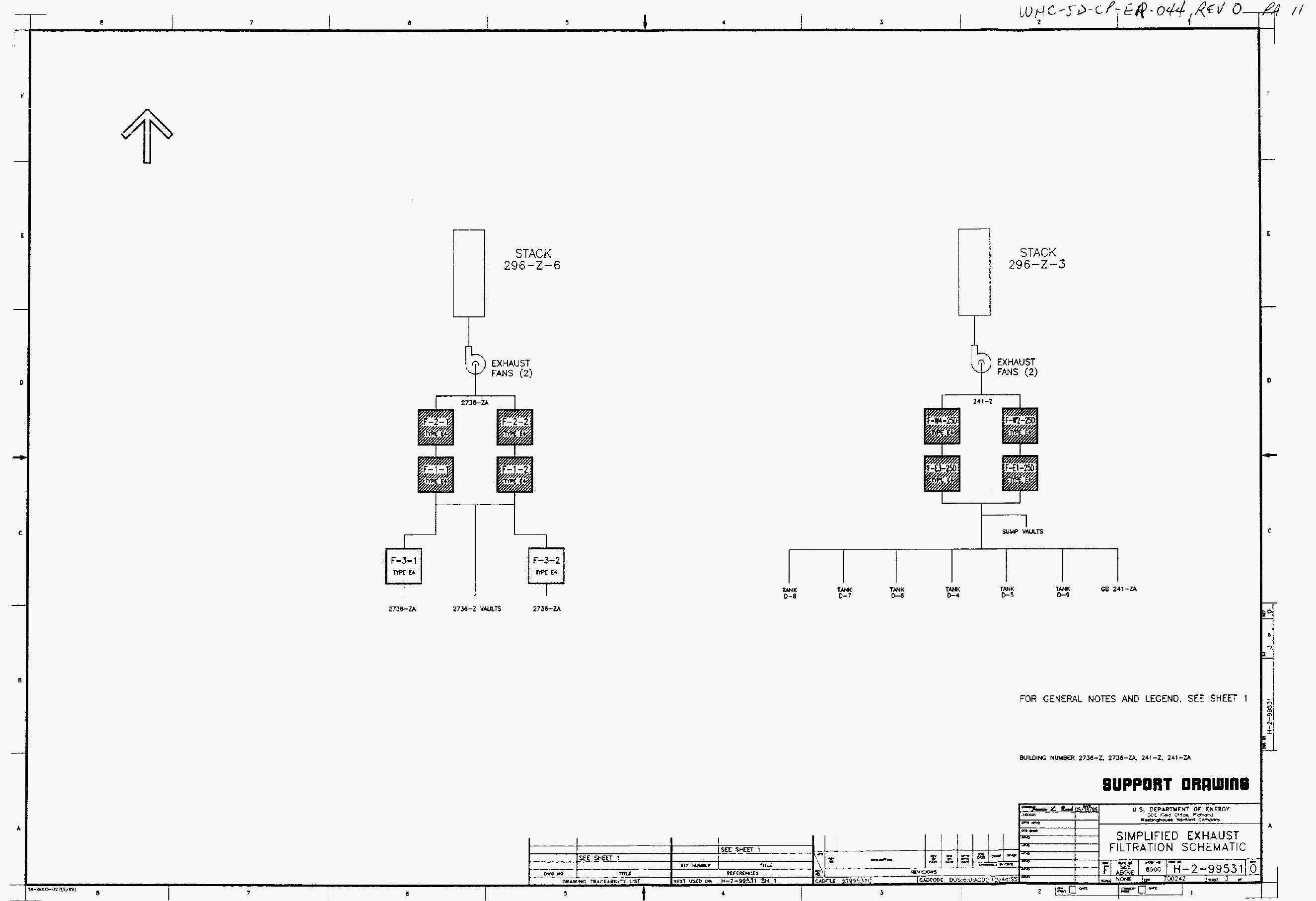



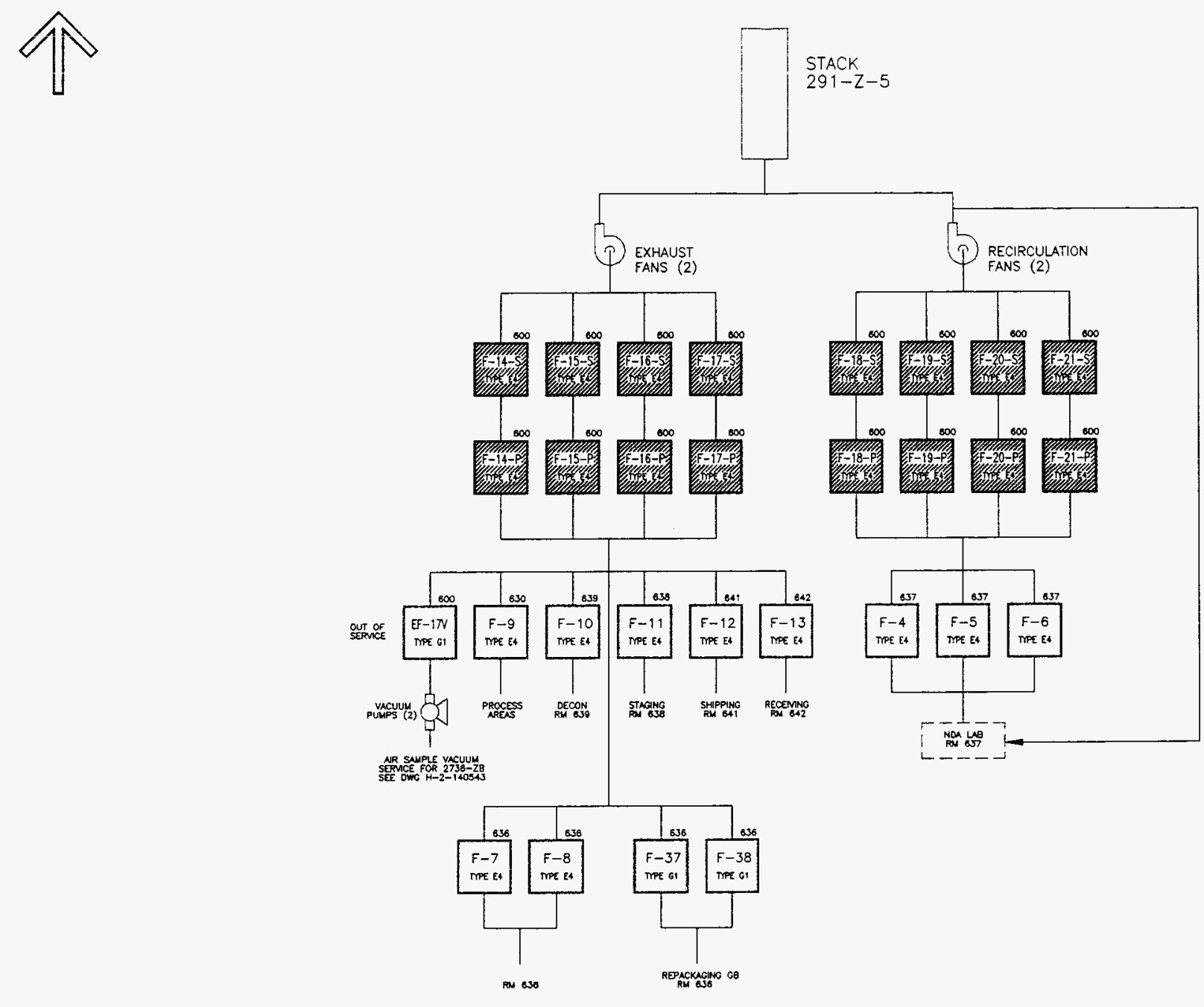

FOR GENERAL NOTES AND LEGEND. SEE SHEET 1

BUPPORT DARUIIA 


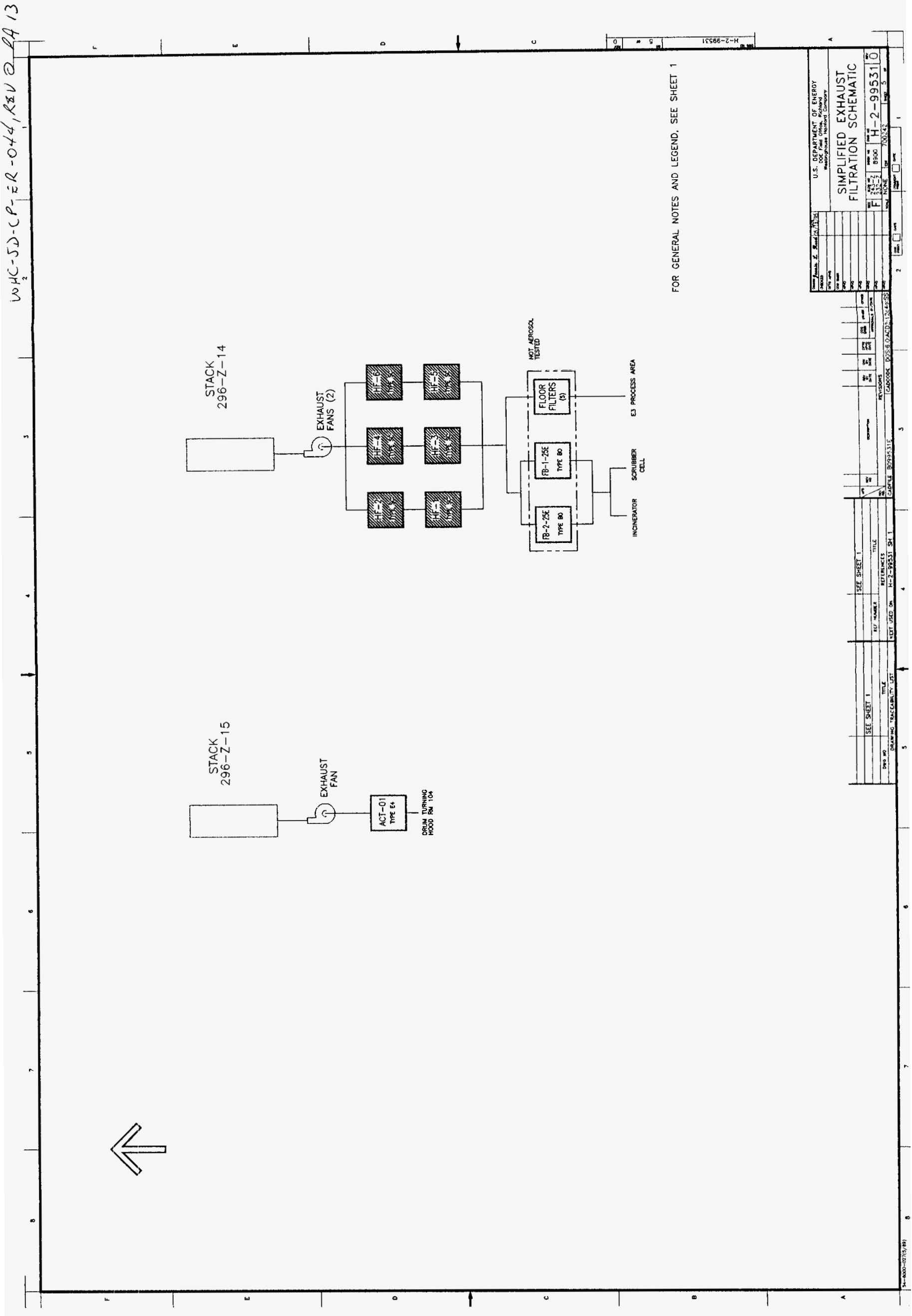


WHC-SD-CP-ER-044 REV. 0

ATTACHMENT 2

page 1 of 5

BYPASS LEAKAGE INSPECTION DEFICIENCIES

\begin{tabular}{|c|c|}
\hline DESCRIPTION & $\mathrm{BLDG} / \mathrm{ROOM}$ \\
\hline LEXAN MANHOLE COVERS (TAPE LOOSE) & $241-2$ \\
\hline NO DEFICIENCIES & $236-Z / 38$ \\
\hline NO DEF ICIENCIES & $243-Z$ \\
\hline $\begin{array}{l}\text { VENTILATORS EXIST BETWEEN ATMOSPHERE AND WALL STUDS OF THE } \\
2736-Z \text { AND } 2736-Z B \text { AIRLOCK }\end{array}$ & 2736-Z COMPLEX \\
\hline $\begin{array}{l}\text { DOOR } 162 \\
\text { (TAPED) }\end{array}$ & $232-z$ \\
\hline \multicolumn{2}{|l|}{ DUCTWORK PAST FINAL FILTERS BLDG 234-5Z } \\
\hline NO DEF ICIENCIES & $\begin{array}{l}\text { STACK MANIFOLD } \\
262\end{array}$ \\
\hline 2 RUST SPOTS MAY PENETRATE ON OUTLET OF FR310 & 262 \\
\hline FLANGE SEPARATED AT WALL CRACK ON OUTLET OF FR309 & 308 \\
\hline TAPE ON OUTLET OF FR311 & 308 \\
\hline FR311 DOS PORTS TAPED & 308 \\
\hline FR312 DOS PORTS TAPED & 308 \\
\hline $\begin{array}{l}\text { FR314 DOS PORTS } \\
\text { TAPED }\end{array}$ & 308 \\
\hline FR315 DOS PORTS TAPED & 308 \\
\hline FR318 DOS PORTS TAPED OR OPEN & 308 \\
\hline DAMPER LINKAGE PENETRATIONS ON ALL FILTER RM OUTLETS & 308 \\
\hline \multicolumn{2}{|l|}{$\begin{array}{l}\text { PRESSURE BOUNDARIES BLDG } 234-5 Z \\
\end{array}$} \\
\hline 2" PENETRATION W/2 PIPES & 180 \\
\hline DOOR 108A (TAPED) & $\mathrm{AL} 108$ \\
\hline $1 / 2^{\prime \prime}$ HOLE & $221 \mathrm{~F}$ \\
\hline DOOR 125 & $171(\mathrm{AL})$ \\
\hline DOOR 129 & CORR $16 \mathrm{AL}$ \\
\hline DOOR 131 & CORR $7 \mathrm{~A} \mathrm{AL}$ \\
\hline
\end{tabular}


WHC-SD-CP-ER-044 REV. 0

ATTACHMENT 2

page 2 of 5

\begin{tabular}{|c|c|}
\hline DESCRIPTION & $\mathrm{BLDG} / \mathrm{ROOM}$ \\
\hline $\begin{array}{l}1 / 2^{\prime \prime} \text { HOLE } \\
\text { EVE LEVEL }\end{array}$ & 262 \\
\hline $\begin{array}{l}\text { 3" FIRE LINE } \\
\text { FLOOR LEVEL }\end{array}$ & 262 \\
\hline $\begin{array}{l}1^{11} \text { CND } \\
\text { FLOOR LEVEL }\end{array}$ & 262 \\
\hline $\begin{array}{l}1 / 2^{\prime \prime} \text { CND } \\
\text { EYE LEVEL }\end{array}$ & 262 \\
\hline $\begin{array}{l}1 / 2 " \text { PIPE } \\
\text { EYE LEVEL }\end{array}$ & 262 \\
\hline $\begin{array}{l}1^{\prime \prime} \text { CND } \\
\text { EYE LEVEL }\end{array}$ & 262 \\
\hline $\begin{array}{l}\text { EXH PLENUM WALL } \\
\text { PENETRATION }\end{array}$ & 262 \\
\hline $\begin{array}{l}1 / 4^{\prime \prime} \text { TUBE } \\
\text { EYE LEVEL }\end{array}$ & 262 \\
\hline $\begin{array}{l}2 \text { EA } 1-2^{*} \\
\text { CND/PIPES } \\
\text { HIGH OVERHEAD }\end{array}$ & 262 \\
\hline $\begin{array}{l}\text { 1" HOLE } \\
\text { EYE LEVEL }\end{array}$ & 262 \\
\hline $\begin{array}{l}\text { LARGE DUCT PENETRATION } \\
\text { OVERHEAD (SUP TO VAULTS) }\end{array}$ & 262 \\
\hline STRUCTURAL STL PENETRATIONS FOR OUTSIDE DUCT SUPPORT & 262 \\
\hline DOOR 563 & $\begin{array}{l}248 \text { AL } \\
\text { COL } 7 \text { OVERPASS }\end{array}$ \\
\hline DOOR 564 & $\begin{array}{l}249 \\
\text { COL } 7 \text { OVERPASS }\end{array}$ \\
\hline $\begin{array}{l}\text { 2" HOLE } \\
\text { (TAPED) } \\
\text { FLOOR LEVEL }\end{array}$ & 308 \\
\hline $\begin{array}{l}7 \text { EA 1/2-1" CND } \\
\text { OVERHEAD }\end{array}$ & 308 \\
\hline $\begin{array}{l}\text { CEILING LEAK } \\
\text { (TAR) }\end{array}$ & 308 \\
\hline
\end{tabular}




\section{WHC-SD-CP-ER-044 REV.0 \\ ATTACHMENT 2 \\ page 3 of 5}

\begin{tabular}{|c|c|}
\hline DESCRIPTION & BLDG/ROOM \\
\hline $\begin{array}{l}1 / 2^{\prime \prime} \text { HOLE } \\
\text { FLOOR LEVEL }\end{array}$ & 308 \\
\hline 4" DRAIN PIPES TO ROOF (TAR) & 308 \\
\hline $\begin{array}{l}1 / 2^{\prime \prime} 90 \# \text { AIR } \\
\text { EYE LEVEL }\end{array}$ & 308 \\
\hline $\begin{array}{l}1 " \text { GLYCOL } \\
\text { FLOOR LEVEL } \\
\end{array}$ & 308 \\
\hline STRUCTURAL STL PENETRATION AT ROOF TRANSITION & 308 \\
\hline $\begin{array}{l}3^{\prime \prime} \text { FIRE PIPE } \\
\text { FLOOR LEVEL }\end{array}$ & 308 \\
\hline $\begin{array}{l}1 " \text { CND } \\
\text { OVERHEAD } \\
\end{array}$ & 308 \\
\hline $\begin{array}{l}1 " \text { PENETRATION W/ } 1 \text { WIRE } \\
\text { EYE LEVEL }\end{array}$ & 320 \\
\hline $\begin{array}{l}1 " 90 \# \text { AIR } \\
\text { OVERHEAD }\end{array}$ & CORR 34 \\
\hline 2 EA $1 / 2^{\prime \prime}$ HOLES & CORR 34 \\
\hline $\begin{array}{l}\text { DOOR } 462 \\
\text { (BAY DOOR) }\end{array}$ & CORR 34 \\
\hline $\begin{array}{l}\text { BATH VENT } \\
\text { (FROM 329) }\end{array}$ & CORR 34 \\
\hline $\begin{array}{l}1 " \text { CND } \\
\text { OVERHEAD }\end{array}$ & $\begin{array}{l}308 \\
(B Y E F-13)\end{array}$ \\
\hline DOOR 280 & $221 \mathrm{~B}$ \\
\hline DOOR 305 & 233 \\
\hline DOOR 306 & 233 \\
\hline DOOR 651 & 2350 \\
\hline $\begin{array}{l}\text { 2" HLAN } \\
\text { THRU UPPER SEC. }\end{array}$ & $194 A$ \\
\hline $\begin{array}{l}1 " \text { CND } \\
\text { ABOVE } 261 \quad \mathrm{AL}\end{array}$ & 260 \\
\hline 1" CND & 260 \\
\hline
\end{tabular}


WHC-SD-CP-ER-044 REV. 0

ATTACHMENT 2

page 4 of 5

\begin{tabular}{|c|c|}
\hline DESCRIPTION & $\mathrm{BLDG} / \mathrm{ROOM}$ \\
\hline $\begin{array}{l}3 " \text { HOLE (TAPED) } \\
\text { FLR LEVEL }\end{array}$ & 260 \\
\hline $2^{\prime \prime}$ CND (TAPED) & 260 \\
\hline OPEN CND CHASE 3 " 4 " CEILING LEVEL & 260 \\
\hline OPEN CND CHASE $3 "-4 "$ CEILING LEVEL & 260 \\
\hline $11 / 2 \& 1^{\prime \prime} \mathrm{CND}$ & 260 \\
\hline $\begin{array}{l}\text { 1" CND, 2-1/8 TUBES IN } 3 \text { "PIPE } \\
\text { (LWBS) }\end{array}$ & 265 \\
\hline 1" CND & 265 \\
\hline 4-1" CND, SAME PENETRATION & 265 \\
\hline 6" ROOF DRAIN & 265 \\
\hline 2-2" CND FROM NEW MCC B & 265 \\
\hline 3" CND, MCC B & 265 \\
\hline CRACKED GROUT AT DRY AIR DUCT PENETRATION & 265 \\
\hline $11 / 2^{\prime \prime} \mathrm{CND}$ & 265 \\
\hline 3" PENETRATION $W / 3$ CND'S & 265 \\
\hline $1 / 2 \mathrm{CND}$ & 265 \\
\hline $\begin{array}{l}2-11 / 2^{\prime \prime} \text { HOLES } \\
\text { OVERHEAD }\end{array}$ & 170 \\
\hline SIDING SEPARATED ABOVE DOOR 126 & 179 \\
\hline $\begin{array}{l}\text { 2" HOLE ABOVE PHONE } \\
\text { HEAD HEIGHT }\end{array}$ & 236 \\
\hline $\begin{array}{l}2 " \text { HOLE } \\
\text { HEAD HEIGHT }\end{array}$ & 154 \\
\hline I" SAN WATER & $235 \mathrm{~B}$ \\
\hline $1^{11} \mathrm{CND}$ & 265 \\
\hline $1 / 2 \& 1^{\prime \prime}$ CND & 265 \\
\hline $\begin{array}{l}5 \text { CND'S } \\
\text { EYE LEVEL }\end{array}$ & 270 \\
\hline $\begin{array}{l}5 \text { CND'S } \\
\text { OVERHEAD }\end{array}$ & 270 \\
\hline
\end{tabular}


WHC-SD-CP-ER-044 REV. 0

ATTACHMENT 2

page 5 of 5

\begin{tabular}{|c|c|}
\hline DESCRIPTION & $\mathrm{BLDG} / \mathrm{ROOM}$ \\
\hline $\begin{array}{l}2-4^{\prime \prime} \text { HOLES } \\
\text { (TAPED) }\end{array}$ & 270 \\
\hline 1" CND & 270 \\
\hline $\begin{array}{l}\text { DOOR } 668 \\
\text { SLIDING (CAULKED) }\end{array}$ & STR 26 \\
\hline
\end{tabular}




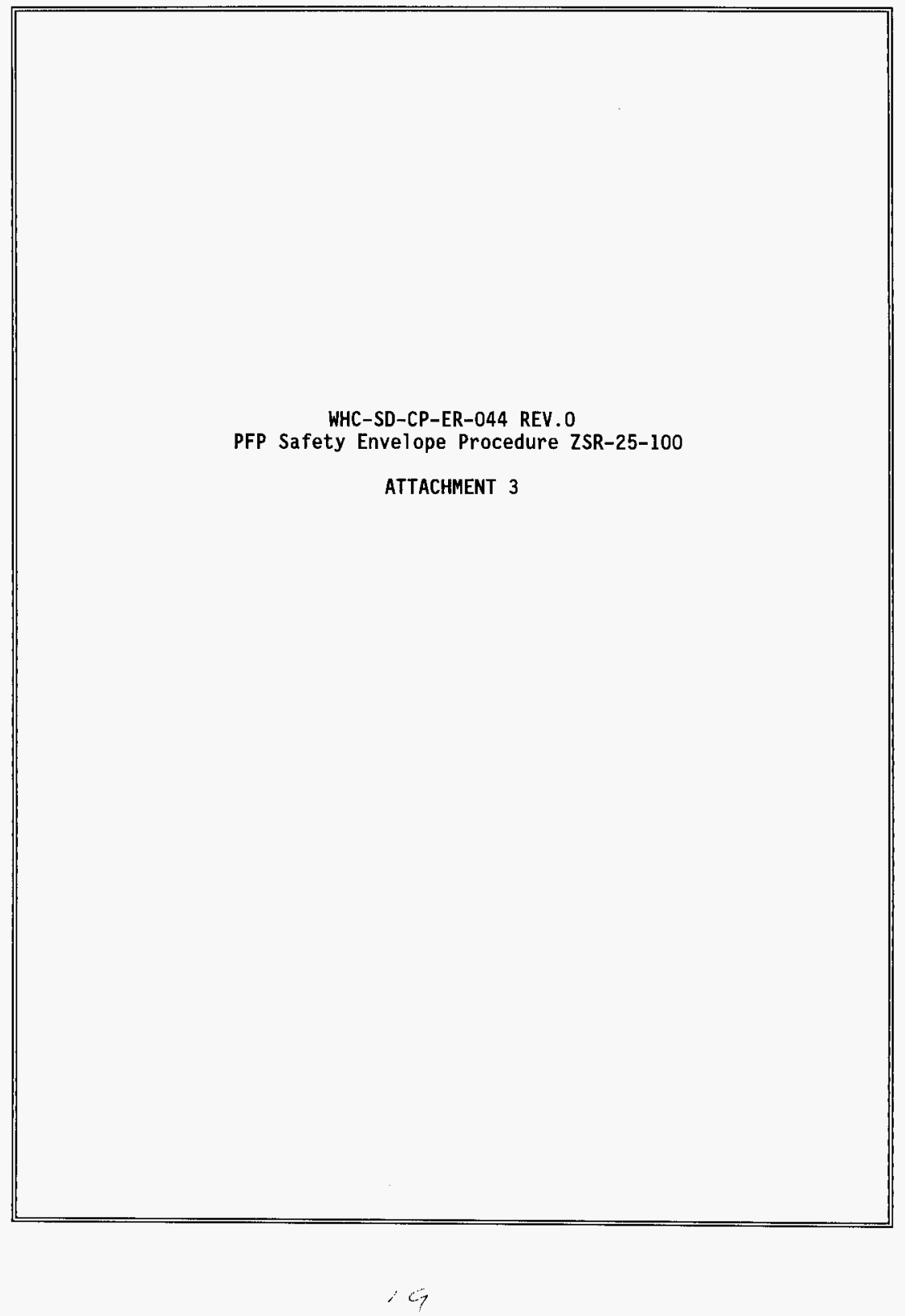




\section{GENERAL}

LSEF

\section{PLUTONIUM FINISHING PLANT}

\section{PROCEDURE}

VENTILATION ZONE BARRIER INSPECTION

SYSTEM 25

\section{ZSR-25-100}

Rev/Mod A-O

Page 1 of 12

Approval Designator S

ISSUE DATE $8 / 28 / 96$

Periodic Review Date $8 / 27 / 98$ 


\begin{tabular}{|c|c|}
\hline $\begin{array}{l}\text { VENTILATION ZONE BARRIER INSPECTION } \\
\text { SYSTEM } 25\end{array}$ & $\begin{array}{l}\text { ZSR }-25-100 \\
\text { REV A-0 } \\
\text { Page } 2 \text { of } 12\end{array}$ \\
\hline
\end{tabular}

\section{REVISION STATUS}

CHANGE PAGE(S) DESCRIPIION

$$
\text { VALIDATION DATE 08/27/96 }
$$

Rev. A-0 All New Procedure
DAF- $96-1031$
DATE $8 / 28 / 96$




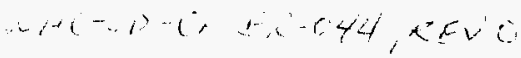

\section{VENTILATION ZONE BARRIER INSPECTION SYSTEM 25}

ZSR-25-100 REV A-O

Page 3 of 12

\section{TABLE OF CONTENTS}

$\underline{\text { SECTION }}$

DESCRIPTION

PAGE

1. INTRODUCTION . . . . . . . . . . . . . . . . . . . . . . . . . . . . . . . 4

1.1 PURPOSE AND SCOPE .......................... . . . 4

2. PERFORMANCE DOCUMENTS . . . . . . . . . . . . . . . . . . . . . 4

3. PERSONNEL REQUIREMENTS . . . . . . . . . . . . . . . . . . . . . . . 4

4. PRECAUTIONS AND LIMITATIONS . . . . . . . . . . . . . . . . . . . . . 4

5. SPECIAL TOOLS, EqUIPMENT, AND MATERIALS . . . . . . . . . . . . . . 4

6. PREREqUISITES . . . . . . . . . . . . . . . . . . . . . . . . . . . 4

7. INSTRUCTIONS . . . . . . . . . . . . . . . . . . . . . . . . . . . . . 5

7.1 Minor Maintenance During Inspections . . . . . . . . . . . . . 5

7.2 Zone Confinement Barrier Inspections . . . . . . . . . . . . . 5

8. RESTORATION . . . . . . . . . . . . . . . . . . . . . . . . . 6

9. TESTING AND ACCEPTANCE . . . . . . . . . . . . . . . . . . . . . . 6

10. DISPOSITION . . . . . . . . . . . . . . . . . . . . . 7

11. BIBLIOGRAPHY . . . . . . . . . . . . . . . . . . . . . . . 7

DATA SHEET 1 - ZONE CONFINEMENT BARRIER INSPECTION . . . . . . . . . . 8

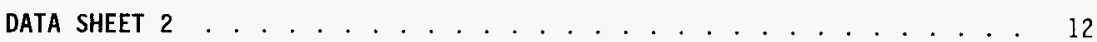




\section{INTRODUCTION}

\subsection{PURPOSE AND SCOPE}

This procedure provides a safe, uniform method of performing inspections of Ventilation Zone confinement barriers at the Plutonium Finishing Plant (PFP). Inspection of zone barriers ensures potential bypass leakage areas are identified. This procedure provides direction for performance of barrier inspection and documents deficiencies.

\section{PERFORMANCE DOCUMENTS}

$\mathrm{H}-2-27650, \mathrm{H}-2-27651, \mathrm{H}-2-27652$, Internal Confinement Bldg. Ventilation Zones

\section{PERSONNEL REQUIREMENTS}

Carpenter(s)

Vent and Balance Power Operator

Radiological Control Technician (RCT), as required.

\section{PRECAUTIONS AND LIMITATIONS}

None

5. SPECIAL TOOLS, EQUIPMENT, AND MATERIALS

Flashlight

\section{PREREQUISITES}

6.1 Potential for radiological contamination EXISTS, REQUEST RCT to perform survey prior to beginning maintenance, or prior to removal of equipment or component from its installed location. 


\section{INSTRUCTIONS}

\subsection{Minor Maintenance During Inspections}

Listed below are minor maintenance activities which may be performed during inspections. Minor maintenance are activities performed without use of additional materials, or by using shop stock materials such as tape or caulking.

Deficiencies observed which require fabrication or procurement of materials or support of additional groups not outlined in this procedure shall be documented on Zone Confinement Barrier Inspection Data Sheet and appropriate JCS package initiated.

Minor maintenance activities which may be performed are as follows;

7.1.1 Taping holes in ductwork and barrier walls.

7.1.2 Sealing conduit and piping penetrations with caulk.

7.1.3 Sealing doors with tape.

\subsection{Zone Confinement Barrier Inspections}

7.2.1 OBTAIN current revision of drawings $\mathrm{H}-2-27650, \mathrm{H}-2-27651$ and H-2-27652.

7.2.2 Visually INSPECT the following barrier walls for leakage paths (i.e. holes, cracks, penetrations or doors not sealed).

- Barrier between 234-5Z Zone 1 and Zones 3 and 3B (first, second and third floors)

- Barrier between 234-5Z Zones 3, 3A and 3B and outside atmosphere and uncontrolled air spaces (tunnels, first, second, third floors and roof) 
7.2.2 - Barrier between 2736-ZB Zone 4 and Zone 2

- Barrier between 2736-ZB and 2736-Z Zone 2 and outside atmosphere

- Barrier between 2736-ZB Zone 3 (Rm 600) and outside atmosphere

- Barrier between 236-Z Zone 3 and outside atmosphere

- Barrier between 241-Z Zone 4 (sump pits) and uncontrolled air space in Building 241-Z

- Barrier between 243-Z Zone 2 and outside atmosphere

- Barrier between 232-Z Zone 3 and outside atmosphere and uncontrolled air spaces (filter room and change room)

7.2.3 Visually INSPECT the following ductwork past the final filters inside 234-5Z for leakage paths (i.e. holes not sealed)

- $\quad$ Outlet duct from filter rooms in Room 308

- Stack Manifold duct in Room 262

\section{RESTORATION}

NOTIFY Shift Manager work is complete.

\section{TESTING AND ACCEPTANCE}

9.1 Minor repairs were satisfactorily completed and appropriate JCS packages initiated.

9.2 No visible bypass leakage through ventilation zone barriers. 


\section{DISPOSITION}

10.1 PIC to record actions taken to resolve deficiencies in comments section of DATA SHEET 2.

10.1.1 If no deficiencies were noted, enter "N/A" and sign data sheet.

10.2 Shift Manager to document acceptance of work on DATA SHEET 2.

\section{BIBLIOGRAPHY}

HSRCM-1, Hanford Site Radiological Control Manual

WHC-IP-1184, Ventilation and Balance Training Program 
VENTILATION ZONE BARRIER INSPECTION SYSTEM 25
ZSR-25-100

REV A-0

Page 8 of 12

\section{DATA SHEET 1 - ZONE CONFINEMENT BARRIER INSPECTION}

Page 1 of 4

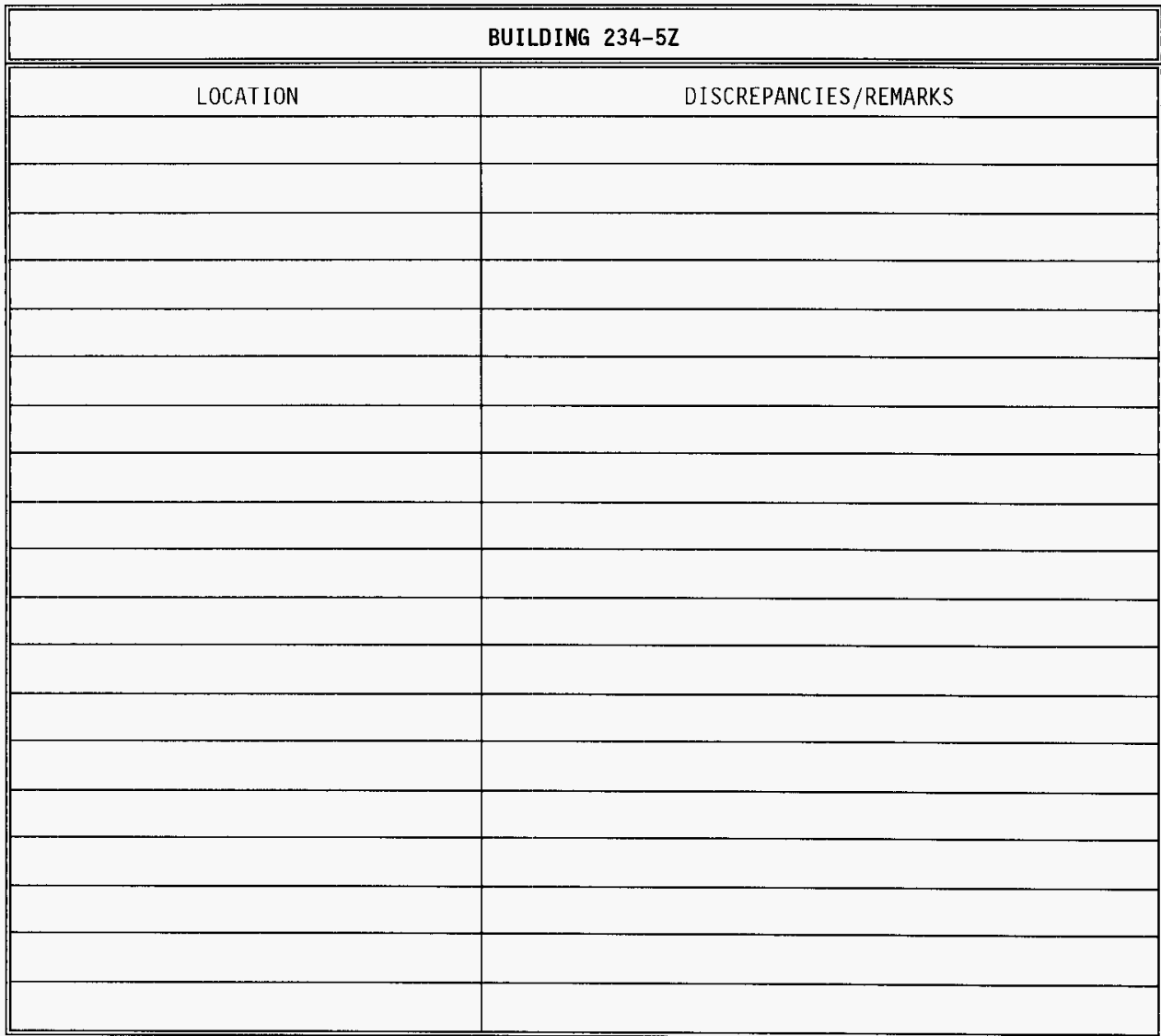

WORK PERFORMED BY:

\begin{tabular}{lll}
\hline INITIALS PRINTED NAME & 1 & \\
SIGNATURE
\end{tabular}

INITIALS PRINTED NAME

SIGNATURE 
VENTILATION ZONE BARRIER INSPECTION

SYSTEM 25
ZSR-25-100

REV A-0

Page 9 of 12

\section{DATA SHEET 1 - ZONE CONFINEMENT BARRIER INSPECTION}

Page 2 of 4

\begin{tabular}{||c|c||}
\hline \multicolumn{2}{|c|}{ BUILDING 2736-ZB AND 2736-Z } \\
\hline \hline LOCATION & DISCREPANCIES/REMARKS \\
\hline & \\
\hline & \\
\hline & \\
\hline \hline & BUILDING 236-Z \\
\hline \hline & \\
\hline & \\
\hline & DISCREPANC IES/REMARKS \\
\hline & \\
\hline & \\
\hline & \\
\hline & \\
\hline
\end{tabular}

WORK PERFORMED BY:

INITIALS

PRINTED NAME

SIGNATURE

INITIALS

PRINTED NAME

SIGNATURE 
W

VENTILATION ZONE BARRIER INSPECTION

SYSTEM 25
ZSR-25-100

REV A-0

Page 10 of 12

\section{DATA SHEET 1 - ZONE CONFINEMENT BARRIER INSPECTION}

Page 3 of 4

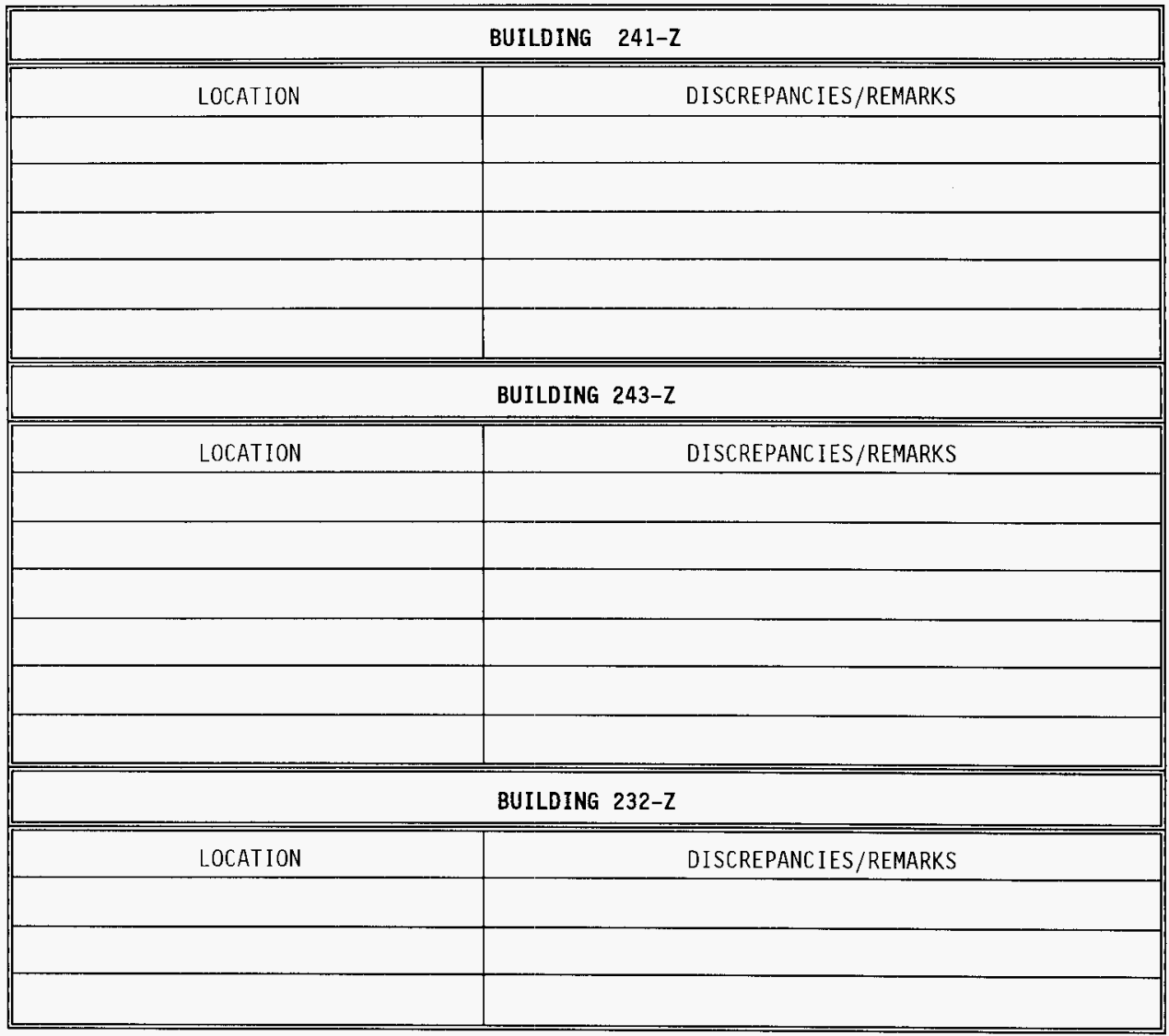

WORK PERFORMED BY:

INITIALS PRINTED NAME

SIGNATURE

INITIALS PRINTED NAME

SIGNATURE 
VENTILATION ZONE BARRIER INSPECTION SYSTEM 25
ZSR-25-100

REV A-0

Page 11 of 12

\section{DATA SHEET 1 - ZONE CONFINEMENT BARRIER INSPECTION}

Page 4 of 4

\begin{tabular}{||c|c||}
\hline \multicolumn{2}{|c|}{ DUCTWORK INSPECTION } \\
\hline \hline LOCATION & DISCREPANCIES/REMARKS \\
\hline & \\
\hline & \\
\hline & \\
\hline & \\
\hline & \\
\hline & \\
\hline & \\
\hline
\end{tabular}

WORK PERFORMED BY:

\begin{tabular}{lll}
\hline INITIALS & PRINTED NAME & SIGNATURE \\
& & \\
\hline INITIALS & PRINTED NAME & SIGNATURE
\end{tabular}




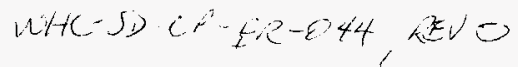

\section{VENTILATION ZONE BARRIER INSPECTION \\ SYSTEM 25}

ZSR-25-100

REV A-0

Page 12 of 12

\section{DATA SHEET 2}

Step

10.1

RECORD below actions taken (e.g., minor maintenance performed or $\mathrm{J}-1 / \mathrm{J}-2$ submitted).

Comments:

PIC:

\begin{tabular}{lll}
\hline & $/$ & \\
\hline INITIALS & PRINTED NAME
\end{tabular}

Step

10.2

Shift Manager acknowledges work on this procedure is complete.

$\begin{array}{lll}1 & \\ \text { INITIALS } & \text { PRINTED NAME }\end{array}$

